\title{
Improved Method for Detection and Classification of Transients and Waveform Distortions Using Sine Fitting Algorithms
}

\author{
T. Radil ${ }^{1}$, P. M. Ramos ${ }^{1,2}$ and A. C. Serra ${ }^{1,2}$ \\ ${ }^{1}$ Instituto de Telecomunicações \\ Av. Rovisco Pais 1, 1049-001 Lisbon, Portugal \\ Phone number: +351218418 489, Fax: +351218418 472, e-mail: tomas.radil@1x.it.pt \\ ${ }^{2}$ Department of Electrical and Computer Engineering, Instituto Superior Técnico, Technical University of Lisbon, \\ Av. Rovisco Pais 1, 1049-001 Lisbon, Portugal \\ e-mail: pedro.ramos@1x.it.pt, acserra@ist.utl.pt
}

\begin{abstract}
In this paper, a method for detection and classification of some power quality disturbances (namely transients and waveform distortions) is presented. The method uses sine fitting algorithms for extraction of the event from the voltage signal and for its classification. The performance of the proposed method is demonstrated by examples of measured events.
\end{abstract}

\section{Key words}

Power quality detection and classification, transients, waveform distortions, sine fitting algorithms.

\section{Introduction}

One of the most common power quality disturbances encountered in power systems are transients and distortions of the voltage waveform (such as harmonic or interharmonic distortions or notching). Monitoring of these disturbances is an important task as these disturbances may have harmful impact on the equipment connected to the power system (e.g., increased levels of harmonic distortion can cause overheating of motors [1]).

The presented method is an improvement of the method presented by the authors [2] and improves the classification stage.

\section{Detection and Classification Method}

The proposed method consists of two stages. In the first stage the presence of potential events is detected. When an event is detected, the method proceeds to the second stage in which the type and the parameters (such as magnitude and duration) of the event are determined. Block diagram of the proposed detection and classification method is shown in Fig. 1. Individual parts are described in the following sub-sections.

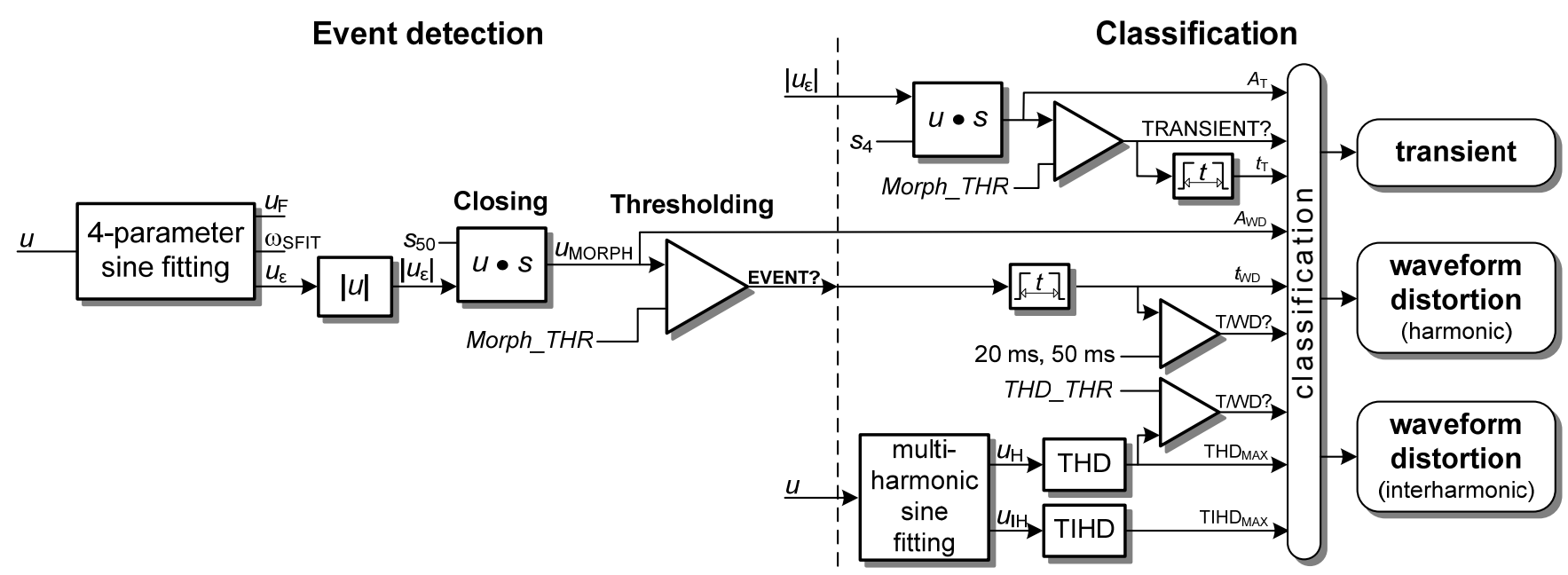

Fig. 1 Block diagram of the proposed detection and classification method 


\section{A. Event detection}

In the detection stage of the proposed method, the power system voltage signal $u$ is processed using the 4-parameter sine fitting algorithm [3]. The 4-parameter sine fitting algorithm is an iterative algorithm that estimates the parameters (the in-phase $A$ and quadrature $B$ component of the amplitude, the frequency $\omega$ and the DC component $C$ ) of a single-tone signal

$$
u_{\mathrm{F}}=A \cos (\omega t)+B \sin (\omega t)+C
$$

The algorithm minimizes the least-square error between the measured signal $u$ and the estimated signal $u_{\mathrm{F}}$. The 4-parameter sine fitting algorithm requires initial estimates of the parameters $A, B, C$ and $\omega$. These are obtained using the 3-parameters sine fitting algorithm [3] (the parameters $A, B$ and $C$ ) with the frequency estimated by the IpDFT algorithm [4].

Estimating the parameters of the fundamental $u_{\mathrm{F}}$ enables to extract the component $u_{\varepsilon}$ that contains potential events

$$
u_{\varepsilon}=u-u_{\mathrm{F}}
$$

The absolute value of the signal $u_{\varepsilon}$ is further processed using mathematical morphology operation closing [5]. The closing operation produces an envelope of the input signal $\left|u_{\varepsilon}\right|$ simplifying detection of potential events

$$
u_{\mathrm{MORPH}}=\left|u_{\varepsilon}\right| \bullet s_{50}
$$

where $s_{50}$ is a so-called structuring element. The events are detected using thresholding and calculating the envelope removes multiples crossings of the threshold level that belong to a single event.

In mathematical morphology, the value of each output sample is based on the value of the corresponding input sample and the samples in its neighbourhood. The size and shape of the neighbourhood are defined by the employed structuring element. The structuring element is a signal containing zeros and ones indicating whether the corresponding samples of the processed signal should be included in the calculation or not. In this case, the structuring element is a signal containing ones, whose length corresponds to $50 \mathrm{~ms}$ ( 2.5 periods of the fundamental at nominal value). For more information on the closing operation and its application in power quality assessment see [6].

The events are detected by thresholding the output signal of the closing operation $u_{\text {MORPH }}$ using a threshold level Morph_THR; i.e., an event is detected when the signal $u_{\mathrm{MORPH}}$ exceeds this threshold level.

\section{B. Classification}

In the classification stage the type and the parameters of the detected event are determined. First, the input voltage $u$ (the part that contains the event) is processed using the non-iterative multiharmonic sine fitting algorithm [7].
The multiharmonic sine fitting algorithm estimates the parameters of $H$ harmonics (in our case $H=40$ ) of the signal $u$ (in-phase component $A_{h}$ and quadrature component $B_{h}$, DC component $\left.u_{\mathrm{DC}}\right)$. Estimating the parameters of individual harmonics allows separating the harmonic $u_{\mathrm{H}}$ and interharmonic $u_{\mathrm{IH}}$ part of the detected event

$$
\begin{gathered}
u_{\varepsilon}=u_{\mathrm{H}}+u_{\mathrm{IH}}, \\
u_{\mathrm{H}}=\sum_{h=2}^{H} A_{h} \cos (h \omega t)+B_{h} \sin (h \omega t), \\
u_{\mathrm{IH}}=u-u_{\mathrm{DC}}-\sum_{h=1}^{H} A_{h} \cos (h \omega t)+B_{h} \sin (h \omega t) .
\end{gathered}
$$

The employed non-iterative multiharmonic sine fitting algorithm is in general less accurate than its iterative version [7]. However, as shown in [8], when an accurate estimate of the signal's frequency is available, the accuracy of the non-iterative algorithm is sufficient for PQ measurements. Also, the non-iterative algorithm is significantly faster. The described method uses the frequency estimate $\omega_{\text {SFIT }}$ provided by the 4-parameter sine fitting algorithm.

The multiharmonic sine fitting is applied only to those parts of the signal $u$ that contain detected events. These parts are further divided into segments that are one period long ( $N$ samples) and the non-iterative multiharmonic sine fitting algorithm is applied to each of these segments. This allows evaluating the evolution of harmonic and interharmonic components in time. In each segment, the total harmonic distortion (THD) and the total interharmonic distortion (TIHD) are calculated

$$
\begin{aligned}
\mathrm{THD} & =\frac{\sqrt{\sum_{h=2}^{H}\left(A_{h}^{2}+B_{h}^{2}\right)}}{\sqrt{A_{1}^{2}+B_{1}^{2}}} \\
\mathrm{TIHD} & =\sqrt{\frac{1}{N} \sum_{n=1}^{N} u_{\mathrm{IH}}^{2}[n]} .
\end{aligned}
$$

An event is classified as a waveform distortion when either of the following conditions is true:

a) its duration exceeds $50 \mathrm{~ms}$ OR

b) its duration is longer than $20 \mathrm{~ms}$ and the THD during the event exceeded the THD_THR threshold.

This means that the proposed method considers an event to be a waveform distortion when the event has a clearly steady-state character (duration longer than 2.5 periods in a $50 \mathrm{~Hz}$ power system) or it is at least one period $(20 \mathrm{~ms})$ long and its harmonic content exceeds a preset threshold level.

Other events are classified as transients; i.e. transients are events that are

a) shorter than one period OR

b) longer than one period, shorter than 2.5 periods and having mostly of interharmonic content. 
The events classified as waveform distortions are further marked as either harmonic or interharmonic depending on the comparison of the maximum values of THD and TIHD. Since in power systems there is always a certain level of both harmonic and interharmonic distortion present, the proposed method, before comparing the THD and TIHD values, subtracts from these maximum values the quiescent THD and TIHD values THD $_{\mathrm{q}}$ and $\mathrm{TIHD}_{\mathrm{q}}$, respectively). This enables to correctly conclude, which of the component (i.e., harmonic or interharmonic) caused the event. The quiescent THD and TIHD are calculated using the non-iterative multiharmonic sine fitting algorithm employing a voltage signal that does not contain any events. A waveform distortion is classified as a harmonic if condition

$$
\max \{T H D\}-T H D_{q}>\max \{T I H D\}-T I H D_{q}
$$

is true and as an interharmonic otherwise.

The events magnitude $A_{\mathrm{WD}}$ is calculated as the maximum value of $u_{\text {MORPH }}$ during the event. The duration of a waveform distortion $t_{\mathrm{WD}}$ is calculated as the time difference between the time instant when the signal $u_{\text {MORPH }}$ crosses the Morph_THR level and the time instant it returns below this level.

In case of transients, further processing is required since the long structuring element $s_{50}$ used to obtain the $u_{\mathrm{MORPH}}$ signal can make several neighbouring transients appear as a single event. In order to separate the potential multiple transients, the signal $\left|u_{\varepsilon}\right|$ is processed using a closing operation once again, this time with a structuring element $s_{4}$ which is only $4 \mathrm{~ms}$ long. The resulting signal is thresholded using the Morph_THR level in order to determine the location in time and the duration $\left(t_{\mathrm{T}}\right)$ of individual transients. The transient's magnitude $A_{\mathrm{T}}$ is calculated as the maximum value of the signal after the closing operation using $s_{4}$.

\section{Measurement Results}

The proposed detection and classification method was tested on events acquired in a single-phase $230 \mathrm{~V} / 50 \mathrm{~Hz}$ power system.

Fig. 2 shows an example of a measured waveform distortion. In Fig. 2a the voltage waveform acquired in the power system containing the event is shown (see Fig. $2 b$ for a detail of the event). Fig. $2 c$ shows the absolute value of the residuals after the 4-parameter sine fitting $\left|u_{\varepsilon}\right|$ and the envelope $u_{\text {MORPH }}$ of this signal obtained using the closing operation. Also shown in this figure is the threshold level Morph_THR used to detect the event. Fig. $2 \mathrm{~d}$ and $2 \mathrm{e}$ show the harmonic $u_{\mathrm{H}}$ and interharmonic $u_{\mathrm{IH}}$ components obtained using the multiharmonic sine fitting algorithm. Using the results of the multiharmonic sine fitting, the THD and TIHD values were calculated. Graph showing the evolution of THD and TIHD during the event is shown in Fig. $2 \mathrm{f}$.
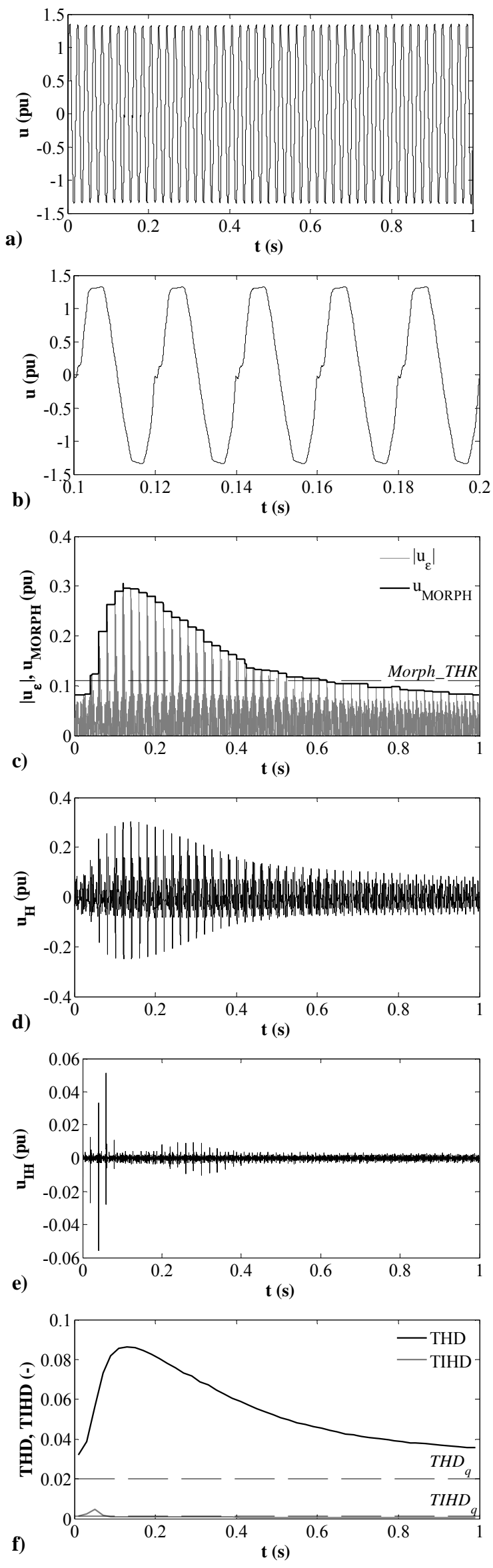

Fig. 2 Example of a measured harmonic waveform distortion and the signals used during the detection and classification. 
The event was classified as a waveform distortion because its duration $t_{\mathrm{WD}}=584.1 \mathrm{~ms}$ exceeded the $50 \mathrm{~ms}$ limit and therefore it's considered to have a steady-state nature. The magnitude of the event is $A_{\mathrm{WD}}=0.305 \mathrm{pu}$. The maximum THD value during the event is 0.087 (i.e., $8.7 \%$ ). Since the increase in THD value is bigger than the increase in TIHD (see Fig. 2f), this waveform distortion was further classified as a harmonic distortion.

Figure 3 depicts another example of a waveform distortion. Also this event is considered to have a steadystate character because its duration exceeds the $50 \mathrm{~ms}$ limit. The magnitude of the shown waveform distortion is $A_{\mathrm{WD}}=1.71 \mathrm{pu}$; its duration is $t_{\mathrm{WD}}=127.87 \mathrm{~ms}$. This waveform distortion was classified as interharmonic because the increase of the TIHD above the quiescent value $\operatorname{TIHD}_{\mathrm{q}}$ was bigger that the increase of the THD above $\mathrm{THD}_{\mathrm{q}}$. The THD during the event reached 0.10 $(10 \%)$; the maximum value of TIHD was $0.11(11 \%)$. In this measurement, the quiescent values were $\mathrm{THD}_{\mathrm{q}}=0.02$ and $\mathrm{TIHD}_{\mathrm{q}}=0.0013$. The interharmonic nature of this waveform distortion can be also seen by comparing the signal $u_{\mathrm{H}}$ (Fig. $3 \mathrm{~d}$ ) and the signal $u_{\mathrm{IH}}$ (Fig. 3e).

Finally, an example of a measured transient is shown in Fig. 4. The event was classified as a transient based on its duration $t_{\mathrm{T}}=1.84 \mathrm{~ms}$. The transient's magnitude is $A_{\mathrm{T}}=0.74$ pu. Fig. $4 \mathrm{c}$ and Fig. $4 \mathrm{~d}$ show that the transient contains both harmonic and interharmonic frequencies. However, as can be seen from Fig. 4e, the increase of the interharmonic content during the event is more significant. Note that the signal $u_{\mathrm{MORPH}}$ shown in Fig. $4 \mathrm{c}$ is the signal after the second closing operation (using the structuring element $s_{4}$ ).

\section{Conclusion}

The proposed method detects and classifies the following three categories of events: i) transients; ii) waveform distortions caused by harmonic frequencies and iii) waveform distortions caused by interharmonic frequencies.

In the event detection stage, the method uses the 4parameter sine fitting algorithm to extract the potential event. When an event is detected, the method uses the non-iterative multiharmonic sine fitting algorithm to separate the harmonic and interharmonic content of the voltage during the event. The classification is based on this information and also on the typical parameters of the considered events [9].

\section{Acknowledgement}

Work sponsored by the Portuguese national research project reference PTDC/EEA-ELC/72875/2006.
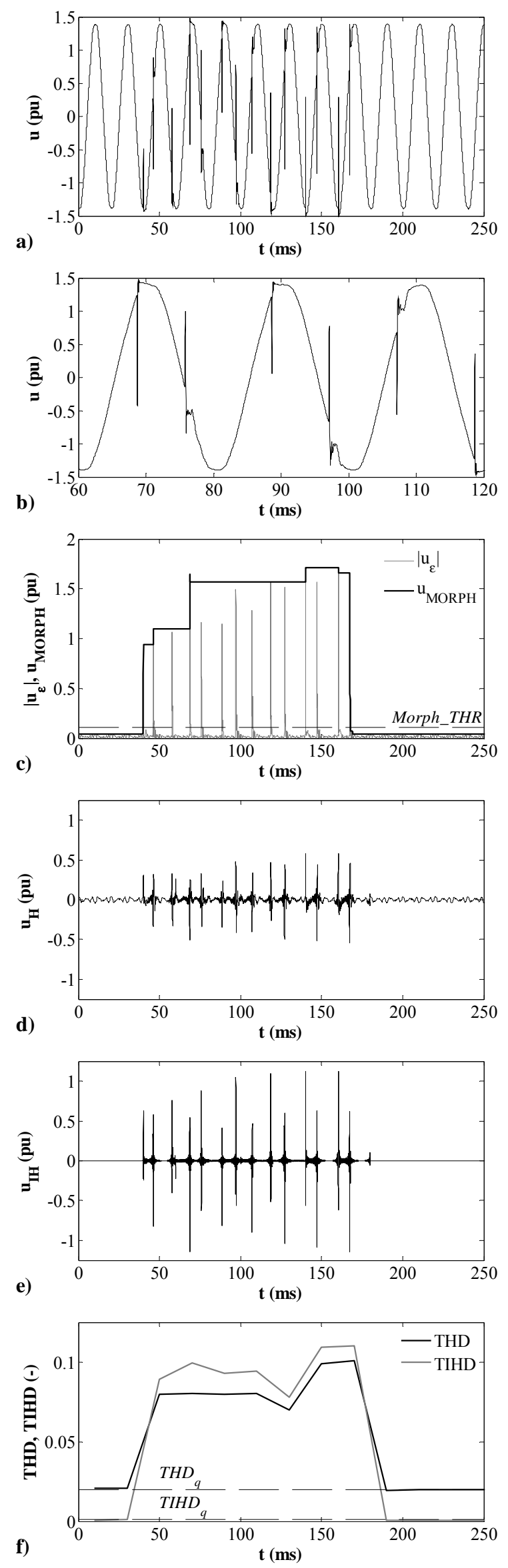

Fig. 3 Example of a measured interharmonic waveform distortion and the signals used during the detection and classification. 

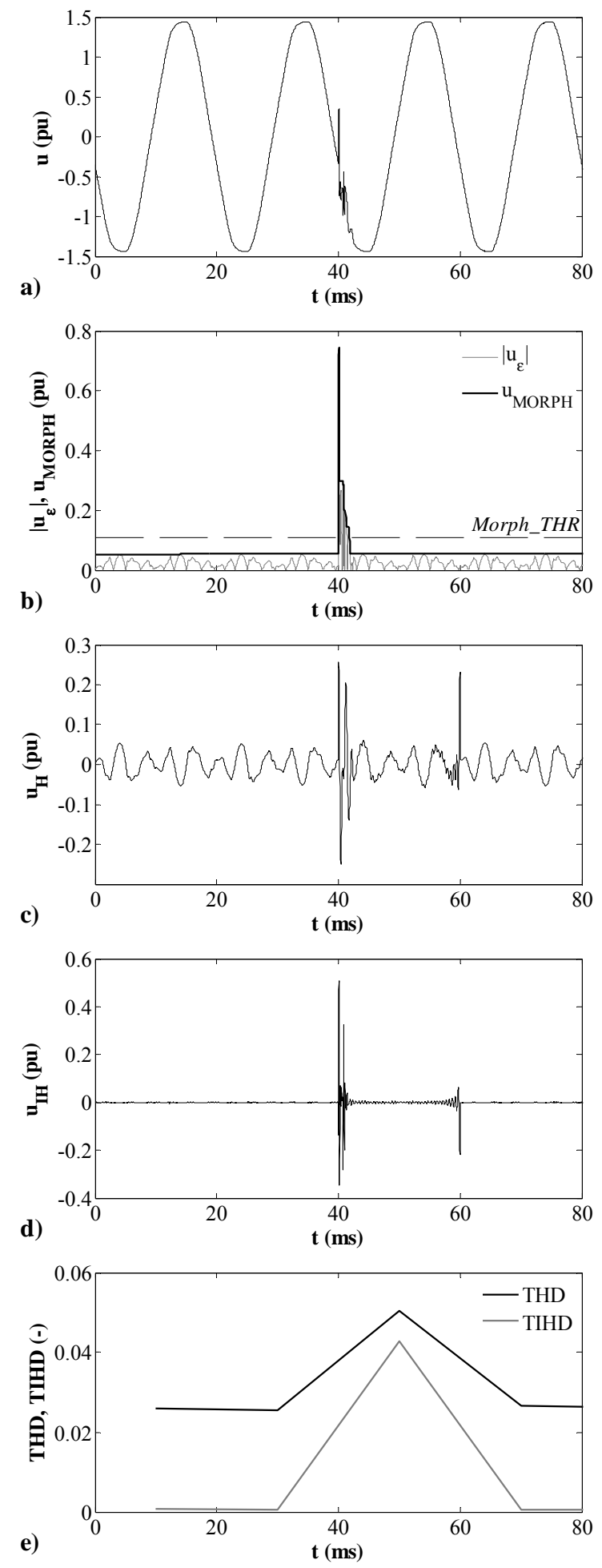

Fig. 4 Example of a measured transient and the signals used during the detection and classification.

\section{References}

[1] A. Baggini, Handbook of Power Quality, Wiley, 2008.

[2] P.M. Ramos, T. Radil, A. C. Serra, "Detection and classification of transients and waveform distortions using an algorithm based on sine-fitting," Proc IMEKO TC4 Symp., Florence, Italy, pp. 323-328, September, 2008.

[3] IEEE Std. 1057-1994 (R2001) - IEEE Standard for Digitizing Waveform Recorders, Sept. 2001.

[4] H. Renders, J. Schoukens, G. Vilain, "HighAccuracy Spectrum Analysis of Sampled Discrete Frequency Signals by Analytical Leakage Compensation", IEEE Trans. Instr. Meas., vol. 33, no. 4, pp. 287 - 292, Dec. 1984.

[5] J. Serra, Image Analysis and Mathematical Morphology, vol. 1, Academic Press, 1982.

[6] T. Radil, P. M. Ramos, F. M. Janeiro, A. C. Serra, "DSP Based Power Quality Analyzer for Detection and Classification of Disturbances in a Single-phase Power System", Metrology and Measurement Systems, Vol. XIV, No. 4, pp. 483 - 494, Dec. 2007.

[7] P. M. Ramos, M. F. da Silva, R. C. Martins, A. M. C. Serra, "Simulation and experimental results of multiharmonic least-squares fitting algorithms applied to periodic signals," IEEE Trans. Instrum. Meas., vol. 55, no. 2, pp. 646-651, Apr. 2006.

[8] T. Radil, P. M. Ramos, A. C. Serra, "Detection and Extraction of Harmonic and Non-harmonic Power Quality Disturbances Using Sine Fitting Methods", Proc International Conf. on Harmonics and Quality of Power - ICHQP, Wollongong, Australia, Sept. 2008.

[9] IEEE Std. 1159-1995 - IEEE Recommended Practice for Monitoring Electric Power Quality, Jun. 1995. 